Cahiers d'études africaines

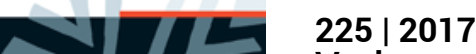

Varia

\title{
LUCARELLI, Carlo. - La huitième vibration
}

\section{Alain Gascon}

\section{OpenEdition}

\section{Journals}

Édition électronique

URL : http://journals.openedition.org/etudesafricaines/20634

DOI : 10.4000/etudesafricaines.20634

ISSN : 1777-5353

Éditeur

Éditions de l'EHESS

\section{Édition imprimée}

Date de publication : 1 avril 2017

Pagination : 191-195

ISBN : 978-2-7132-2688-5

ISSN : 0008-0055

\section{Référence électronique}

Alain Gascon, «LucARELLI, Carlo. - La huitième vibration », Cahiers d'études africaines [En ligne], 225 |

2017, mis en ligne le 01 avril 2017, consulté le 24 septembre 2020. URL : http://

journals.openedition.org/etudesafricaines/20634; DOI : https://doi.org/10.4000/etudesafricaines. 20634

Ce document a été généré automatiquement le 24 septembre 2020.

(c) Cahiers d'Études africaines 


\title{
LUCARELLI, Carlo. - La huitième vibration
}

\author{
Alain Gascon
}

\section{RÉFÉRENCE}

LuCARELLI, Carlo. - La huitième vibration. Trad. par S. Quadruppani, Paris, Métailié, 2010, $413 \mathrm{p}$.

1 Il n'est pas fréquent de recenser dans un même texte une œuvre de fiction, le roman de C. Lucarelli, et un livre d'histoire. J. Miran, l'historien, a compulsé les archives en Érythrée, à Londres, Rome et Paris, constitué une importante bibliographie et mené des enquêtes approfondies dans le port de Massawa en Érythrée. Son travail s'appuie sur un corpus documentaire en anglais, italien, français, allemand, en arabe et dans les langues éthio-érythréennes (tigré, tigrigna, amharique). C. Lucarelli est un auteur italien renommé de romans policiers à fort engagement social et politique qu'on pourrait comparer à Didier Daeninckx en France. Le livre est traduit de l'italien par S. Quadrupanni qui a su remarquablement rendre les nuances et les particularités des dialectes italiens et des langues éthio-érythréennes ${ }^{29}$. L'action de La huitième vibration se déroule à Massawa avant la bataille d'Adwa (1896) puis se termine par une évocation lyrique de la défaite italienne, alors que J. Miran étudie le temps long des mutations culturales et religieuses survenues dans la société « cosmopolite des citoyens de la mer Rouge » de la seconde moitié du xix ${ }^{e}$ siècle aux vingt premières années du $\mathrm{xx}^{\mathrm{e}}$ siècle. Ce port redevint, avec Port-Soudan, Jiddah, Aden, l'une des portes des rives de la mer Rouge à la fin de la période ottomane (1557-1865) avant même l'inauguration du canal de Suez (1869). L'essor de Massawa, «le Zanzibar de la mer Rouge»(p.65), sous administration égyptienne en 1865 , se poursuivit après la prise de possession par l'Italie en 1885. La plus grande partie de l'action du roman de C. Lucarelli s'inscrit donc dans la période étudiée par J. Miran et même s'il ne peut être mis sur le même plan que les travaux universitaires tels que ceux d'Haggai Erlich $^{30}$ dont la lecture est 
recommandée à qui veut comprendre la période où Européens, Égyptiens se disputaient les rivages. Soucieux de vraisemblance, le romancier a rassemblé une importante documentation qui lui a évité tout anachronisme et toute erreur grave dans ses transcriptions des langues locales ${ }^{31}$. C. Lucarelli complète donc le travail érudit de l'historien car, à partir d'une description célinienne du microcosme colonial enfermé dans les îles madréporiques de Massawa, il conforte la plupart de ses analyses. Red Sea Citizens relativise fortement l'idée d'une civilisation "thalassocratique » de la mer Rouge qui, à l'instar des ports swahili de l'océan Indien, serait étrangère aux hinterlands. L'intrigue de La huitième vibration dépeint des Italiens qui, retranchés dans les îles et leurs certitudes, sont espionnés et instrumentalisés par les «indigènes »dont ils ne peuvent se passer - et qui les attirent irrésistiblement sur le continent dans le piège d'Adwa.

2 Je mets de côté, un temps, le roman de C. Lucarelli - que je reprendrai en conclusion pour me consacrer au travail ambitieux de J. Miran. Il renouvelle nos connaissances sur l'histoire de Massawa et de ses relations avec le Piémont et les hautes terres éthioérythréennes et avec le Yémen, le Hedjaz et l'Inde. La lecture du livre est facilitée par un glossaire très complet, un index très utile et des photographies de notables, de caravanes, de scènes de marché et de rue, de cérémonies confrériques et de maisons et de mosquées. Sur la carte de l'hinterland $\left(\mathrm{n}^{\circ} 4, \mathrm{p} .48\right)$, à bien trop petite échelle, toutefois, on devine que la gradation des gris coïncide avec les courbes de niveau, mais lesquelles? C'est dommage qu'on ne le sache pas car, comme l'écrit fort justement l'auteur, à Massawa, héritier du port aksumite de Zula, les hautes terres éthioérythréennes surplombent, de plus de 2000 mètres, la tihama, la plaine littorale. L'escalade n'est certes pas facile, mais en quelques étapes jalonnées de sources, on atteignait les plateaux sans avoir à traverser des semaines de désert aride sous la menace des razzias des nomades. On aurait aussi aimé trouver sur cette carte, même de façon sommaire, la répartition des populations saho, tigré... qui, de plus en plus nombreuses tout au long de la période, ont gagné la conurbation de Massawa : à savoir les îles de Massawa et de Tewalet (Taulud), les péninsules d'Abd el-Qader et de Gherar et les bourgs de Hergigo, Emkullo et Hemtulo situés sur la terre ferme.

3 J. Miran a adopté un plan rationnel dont les cinq grandes parties, encadrées par une introduction et une conclusion, s'articulent bien entre elles. L'énoncé des titres est particulièrement heureux : ils annoncent de façon très claire la question dont traite la partie et je les citerai afin de résumer la démarche de l'auteur. L'introduction : « Facing the Land, Facing the Sea » (pp. 1-32) et la $1^{\text {re }}$ partie : « Making a Region between the Sea and the Mountain» (pp.33-64) brossent un tableau historique, anthropologique, linguistique et géopolitique de Massawa un port d'interface, un complexe culturel : « to mediate between multiple commercial spheres and circuits » (p.3) entre les premières décennies $d u \mathrm{XIX}^{\mathrm{e}}$ siècle et la première décennie du $\mathrm{xx}^{\mathrm{e}}$ siècle et un lieu de métissage "par excellence» (p.17). J. Miran insiste sur l'émergence du pouvoir des naib de Hergigo - recrutés parmi les Balaw d'origine saho et béja - et de leurs familles qui contrôlaient l'importation des armes à feu et le commerce des esclaves. D'abord fonctionnaires de l'empire Ottoman, ils s'émancipèrent peu à peu si bien que les Égyptiens, puis les Italiens les enrôlèrent dans leur encadrement administratif sans, toutefois, réduire leur autonomie.

4 Les parties $n^{\circ} 2$ : «On Camels and Boats» (pp. 65-111) et $n^{\circ} 3:$ " Connecting Sea and Land» (pp.112-165) retracent comment les marchands ont fait de Massawa, avant 
même l'ouverture du canal de Suez, le «Zanzibar de la mer Rouge » (p. 65), si bien qu'il a dépassé Moka et s'est hissé au rang d'Aden et de Jeddah. L'auteur met en avant le rôle des Hadramawi qui ont rejoint les Arabes et les Indiens et maintenu un système dualiste de navigation : les cargos à vapeur n'ont pas éliminé les embarcations à voile qui utilisent l'alternance des vents de mousson. Sur le continent, on distinguait deux grands courants caravaniers partant et aboutissant à Massawa. La piste, de direction est-ouest, des caravaniers locuteurs du tigré rejoignait, par Kärän et les Bogos, Kassala et le Soudan conquis par les Égyptiens. Par la grande route caravanière nord-sud, parcourue par les Jeberti (des commerçants éthiopiens musulmans), parvenaient sur le littoral les produits agricoles des basses terres du Semhar, proches du port, puis des hautes terres au nord du Nil bleu (céréales, cuirs et peaux) et des royaumes oromo du Sud-Ouest, notamment du café et aussi des esclaves (de $37 \%$ du trafic en 1842 à $12 \%$ en 1861). Pendant la période égyptienne, l'afflux des populations environnantes a gonflé les effectifs des villages de Hergigo, Emkullo et Hetmulo qui accédèrent au rang d'agglomérations citadines dans la conurbation de Massawa qu'une chaussée-digue réunissait désormais les îles à la terre ferme. Ouvriers yéménites et esclaves oromo libérés étaient venus s'engager dans les travaux de manutentions des navires. L'auteur note que les familles des " armateurs », des courtiers et des entrepreneurs caravaniers issues de l'Arabie, d'Hadramawt/Hadramaout, d'Inde (les Banians dans la finance), à mesure qu'elles gagnaient en richesse et notoriété, s'établissaient d'abord sur la terre ferme puis dans l'île où l'espace était pourtant limité : en 1840, la moitié des terrains y était occupée par des tombes, notamment celles de « saints » liés aux confréries.

La $4^{\text {e }}$ partie (pp. 166-216) s'intitule fort justement: "A Sacred Muslim Island » et introduit la dernière partie, $\mathrm{n}^{\circ} 5$ : «Being Massawan" (pp. 217-267) où J. Miran entreprend une approche très fine de l'identité des habitants de la ville-port, venue «de la terre et de la mer». Il identifie un réveil de l'islam, après 1850, dans les dernières années de l'empire Ottoman dont les promoteurs étaient originaires de la péninsule Arabique (la Mecque), de la vallée du Nil, d'Égypte et du Soudan. Des familles " saintes » migrèrent, répandant sur les rives africaines, le wahhabisme, le mahdisme, le soufisme et les grandes confréries Qadiriyia, Shadhiyya, Ahmadiyya, Tijaniyya et Khatmiya (sous l'influence des Mirghani). En même temps, les peuples agro-éleveurs et locuteurs du tigré des basses terres du Nord, Nara ${ }^{32}$, Kunama et Bilen abandonnèrent le christianisme et/ou le paganisme pour l'islam. Le gouverneur d'Érythrée, Martini, s'appuya sur la Khatmiyya et la soutint contre les musulmans qui s'indignaient des cérémonies accompagnées de danses, de tambours et d'applaudissements. Ces conflits opposaient les "grandes familles» de Massawa aux «nouveaux riches» (p. 240) qui recherchaient le prestige d'une origine " arabe » par le vêtement, l'usage de la langue arabe, l'enseignement dans les madrasa, la participation aux confréries et aux waqf, l'achat d'un terrain sur la terre ferme puis dans l'île et par la construction d'une maison à étage en pierre ornée de balcons, de sculptures, de moucharabieh et d'inscriptions louant Allah et son Prophète. Ce processus d'acquisition de la citoyenneté de Massawa, utilisée comme une marque déposée (Trade Mark, p. 251) entamé sous la tutelle égyptienne se poursuivit sous la colonisation italienne. J. Miran, par un travail très précis, nous entraîne dans les stratégies matrimoniales complexes à la fois endogamiques (Hadramawi, Arabes) et exogamiques: "Massawa is one big family » (p. 257) déclara un informateur. Et l'auteur de résumer judicieusement ces parties: "In other words, in this period "being Massawan" meant being urban and being Arab » (p. 267). 
6 La courte conclusion (pp. 268-279) est fort bien résumée par son sous-titre : "Constructing and Deconstructing a Red Sea Society ». Même si le contexte historique et géopolitique de la mer Rouge entre 1850 et 1920 est bien différent de celui de l'Europe des XVIII $^{\mathrm{e}}$ et $\mathrm{XIX}^{\mathrm{e}}$ siècles, avec, de plus, le poids de la colonisation, lorsque l'on lit les analyses de J. Miran sur la formation de la société marchande de la mer Rouge, vient à l'esprit la référence à l'éthique du capitalisme de M. Weber. Le protestantisme prendrait en quelque sorte "la place » de l'islam soufique, rigoureux et confrérique, porté par les marchands et les marins venus du Soudan, d'Égypte, d'Arabie et d'Hadramwt. Peu d'Italiens, hormis quelques chercheurs (Odorizzi) et le gouverneur Martini ont perçu l'importance des mutations socio-culturelles à Massawa comme le montre Lucarelli. Dans La huitième vibration, ils vivent dans une île à l'intérieur de l'île, un huis clos, où s'assouvissent leurs querelles, leurs rivalités, leurs mesquineries et où se perpétuent des meurtres... à l'abri, croient-ils, des regards de l'Italie et du continent. Mais l'enquête policière les rattrape, les espions de Menilek les épient et connaissent non seulement le détail de plans militaires mais jusqu'à leurs coucheries les plus intimes. Le roman complète également le livre de J. Miran quand il aborde la question de la formation nationale de l'Éthiopie et l'Italie. Après une longue période de désunion et de fragmentation, l'Italie sous la direction de la maison de Savoie et l'Éthiopie sous la poigne des rois des rois "modernisateurs", retrouvent leur unité nationale et territoriale par la guerre. Le soldat qui, dans le roman, échappe à la catastrophe d'Adwa est un humble paysan, envoyé en Afrique par accident. Il cultive le champ d'une Éthiopienne veuve qu'il a rencontrée après la bataille et qui l'accepte dans sa hutte.

7 Red Sea Citizens apporte de précieuses informations sur l'insertion de Massawa dans l'Érythrée coloniale et postcoloniale. La ville-port n'en fut la capitale que de 1890 à 1899 avant l'établissement des Italiens à Asmära. Il en résulta un afflux de populations chrétiennes des hautes terres renforcées par l'arrivée de colons italiens et par les conversions des missions catholiques et protestantes, alors qu'en 1841, le consul de France avait soulevé une opposition résolue à l'installation d'une chapelle. Le mouvement des Arabes de Massawa en 1940-1950 ne réussit pas à apaiser les rivalités entre confréries dans la ville et dans l'ex-colonie. La Ligue musulmane de l'Ouest rompit avec la Ligue musulmane et approuva la fédération avec l'Éthiopie comme les chrétiens monophysites soutenus par l'Église éthiopienne ${ }^{33}$. Les «Arabes » de Massawa aidèrent à accréditer auprès des pays arabes l'idée que l'Érythrée appartenait au monde arabe et qu'ils devaient soutenir le Front de libération d'Érythrée, animé à ses débuts, pourtant, par les Beni-Amer des basses terres de l'Ouest. Quand les autorités de l'Érythrée indépendante distribuèrent des cartes d'identité, beaucoup d'habitants de Massawa remplirent la case d'appartenance "ethno-linguistique » par la mention «Arabe » ou « Massawa »! J. Miran évoque aussi le déclin de l'activité portuaire après l'essor constant du XIX ${ }^{e}$ siècle, puis un repli consécutif à l'agression et à l'occupation italienne de l'Éthiopie et enfin, une reprise pendant la fédération avec l'Éthiopie (1952-1962) ${ }^{34}$. En 1897, commençaient les travaux du chemin de fer de Djibouti à Addis Abäba qui atteignit Dirré Dawa en 1902 puis la capitale en 1917. Au grand dam de Massawa, Djibouti traitait de 70 à $80 \%$ du trafic éthiopien avant la guerre. Au début des années 1960, Asäb, port érythréen désormais accessible par la route supplanta Massawa et même Djibouti jusqu'au conflit éthio-érythréen de 1998 qui provoqua le report sur Djibouti du commerce international éthiopien. 
Le livre de J.Miran apporte sur une période, une région et une ville-port des connaissances nouvelles dans beaucoup de domaines: le commerce, les routes maritimes et terrestres, les marchands, leurs origines, leurs alliances, leurs généalogies et leurs familles, sur les migrations, les mutations culturelles et religieuses d'une des villes-clés de la mer Rouge. Il réussit à embrasser un domaine, centré sur la mer Rouge, qui s'étend de l'Europe à la Méditerranée, à l'Égypte, au Soudan, à la Corne de l'Afrique, à la péninsule Arabique et à l'océan Indien. L'auteur maîtrise ce jeu d'échelles emboittées et il est particulièrement à l'aise pour dénouer le fil des alliances matrimoniales, commerciales et confrériques. Il nous donne à lire un tableau vivant, écrit d'un style alerte en dépit d'une matière très complexe. Son ouvrage peut être donné en exemple aux jeunes chercheurs. Il intéressera tous ceux qui connaissent, et qui ne connaîtraient pas, la mer Rouge, ses rivages et son peuplement. Il rompt aussi avec une tradition, maintenant abandonnée, d'étudier et de considérer les basses terres de la Corne de l'Afrique et leurs populations établies sur ces marges, comme simples couloirs de passage vers les peuples de culture écrite et plus nobles des hautes terres. Le livre de J. Miran remet enfin en question, après bien d'autres, l'idée reçue qui veut qu'en Afrique on soit «incapable » de former des élites et qu'on doive les importer d'Europe, des États-Unis ou du Moyen-Orient. Massawa fut aux $\mathrm{XIX}^{\mathrm{e}}$ et $\mathrm{Xx}^{\mathrm{e}}$ siècles un atelier où « indigènes » et migrants devinrent des citoyens de la mer Rouge.

Le lecteur de l'ouvrage universitaire érudit de Jonathan Miran ${ }^{35}$ suivra avec profit les péripéties de l'enquête policière à Massawa et la description de la bataille d'Adwa, racontées avec un talent consommé par Carlo Lucarelli, comme on se plonge dans l'Afrique coloniale avec Bardamu dans Voyage au bout de la nuit ou avec Simenon dans Coup de lune et dans $45^{\circ}$ à l'ombre. On peut, aussi, passer des moiteurs de la Huitième vibration à l'exposé clair et précis de Red Sea Citizens.

\section{NOTES}

29. A. GASCON \& A. TuRco, "Carlo Lucarelli, La huitième vibration », POUNT, 6, 2012, pp. 187-199.

30. Ras Alula and the Scramble for Africa. A Political Biography : Ethiopia and Eritrea 1875-1897, Lawrenceville, The Red Sea Press, 1996.

31. A. GASCON \& A. TURCO (2012), op. cit.

32. On surnommait les Nara, Bariya (c'est-à-dire esclave en amharique et en tigrigna), car ils subissaient les razzias des esclavagistes chrétiens des hautes terres et des musulmans des basses terres. Leur conversion à l'islam les mettait dorénavant à l'abri des raids musulmans.

33. T. N EGASH, Eritrea and Ethiopia: The Federal Experience, Uppsala, Nordiska Afrikainstitutet-New Brunswick, Transaction, 1997.

34. I. TADDIA, L'Eritrea-colonia 1890-1952 : paesaggi, strutture, uomini del colonialismo, Milan, 1986. 
35. Formé initialement à l'INALCO, il est maintenant professeur assistant de civilisation islamique dans le Département des arts libéraux de l'Université Western Washington. 